\title{
LESÃO DE ISQUEMIA E REPERFUSÃO APÓS CLAMPAGEM CONTÍNUA OU INTERMITENTE DO PEDÍCULO HEPÁTICO EM COELHOS
}

\author{
Ischemia/reperfusion injury after continuous or intermittent hepatic pedicle clamping in rabbits \\ André Luis Ramires SEABRA ${ }^{1}$, Paulo Roberto SAVASSI-ROCHA², Anilton César VASCONCELOS ${ }^{2}$, \\ Agnaldo Soares LIMA ${ }^{2}$, Kelly Cristine Lacerda RODRIGUES ${ }^{2}$, Herbert Motta de ALMEIDA $^{1}$
}

Trabalho realizado na ${ }^{1}$ Faculdade de Medicina da Universidade de Ciências da Saúde de Alagoas, Maceió, AL, e Faculdade de Medicina da Universidade Federal de Minas Gerais, Belo Horizonte, MG, Brasil

DESCRITORES - Isquemia/cirurgia. Traumatismo por reperfusão. Fígado/cirurgia. Apoptose.
RESUMO - Racional - O controle do sangramento na hepatectomia é um desafio para os cirurgiões. A clampagem do pedículo hepático é manobra cirúrgica que pode promover redução do sangramento, mas provoca isquemia hepatocelular. Isso, junto com a reperfusão depois que a clampagem termina, leva à lesão de isquemia e reperfusão. Objetivo - Examinar os efeitos da lesão de isquemia e reperfusão no fígado após clampagem contínua e intermitente do pedículo hepático, usando a quantificação de apoptose como ferramenta. Método - Vinte coelhos New Zealand foram divididos em grupos 1 (controle), 2 (60 minutos de isquemia contínua) e 3 (60 minutos de isquemia intermitente alternando 12 minutos de isquemia e três minutos de reperfusão). Biópsias hepáticas foram colhidas antes e ao fim da isquemia e após seis horas de reperfusão, quando os animais eram sacrificados. Os fragmentos obtidos foram submetidos à análise histológica e histoquímica (reação de Tunel). Campos microscópicos foram analisados para caracterização e quantificação de apoptose. Resultados - A isquemia levou à elevação do índice apoptótico em ambos os grupos experimentais em relação aos controles, mas similar entre eles. Depois da reperfusão os índices voltaram aos valores iniciais. Conclusão - A clampagem do pedículo hepático, tanto contínua quanto intermitente, induz a apoptose em células hepáticas de modo igual.

\author{
Correspondência: \\ André Luis Ramires Seabra, \\ e-mail: andre@seabras.net \\ Fonte de financiamento: Fundação de \\ Amparo à Pesquisa de Minas Gerais - \\ FAPEMIG (http://www.fapemig.br) \\ Conflito de interesses: não há \\ Recebido para publicação: \\ Aceito para publicação:
}

HEADINGS - Ischemia/surgery. Reperfusion injury. Liver/surgery. Apoptosis
ABSTRACT - Background - The control of bleeding in hepatectomy is a challenge for surgeons. The hepatic pedicle clamping is a surgical maneuver that can provide reduction in bleeding, but it provokes a hepatocellular suffering. This, along with reperfusion after the clamping finishes, leads to an injury known as ischemia/reperfusion injury. Aim - To examine the effects of the ischemia/reperfusion injury on the liver after continuous and intermittent hepatic pedicle clamping in an animal model, using the quantification of apoptosis for evaluation. Method - Twenty New Zealand rabbits were assigned to groups 1 (control), 2 (60 minutes of continuous ischemia) and 3 (60 minutes of intermittent ischemia alternating 12 minutes of ischemia and three minutes of reperfusion). Liver biopsies were collected before ischemia, at its end and after six hours of reperfusion, when the animals were killed. The liver fragments were subjected to histological analysis (paraffinization and hematoxilin-eosin staining) and histochemical (Tunel reaction). Microscope fields of view were scanned for characterization and quantification of apoptosis. Results - Ischemia led to an increased apoptotic index in both experimental groups in comparison to controls, but similarly between them. After the reperfusion, the indexes returned to baseline values. Conclusion - Clamping of the hepatic pedicle, either continuous or intermittent, induces apoptosis in liver cells in a similar way.

\section{INTRODUÇÃO}

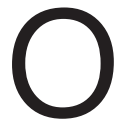
controle do sangramento na cirurgia hepática continua sendo grande desafio para os cirurgiões. As técnicas de controle incluem a clampagem do pedículo hepático proposta por Pringle ${ }^{24}$ e a exclusão vascular total, entre outros. O objetivo comum é reduzir a hemorragia do parênquima hepático, permitindo ao cirurgião realizar o procedimento sem sangramentos que possam interferir na morbidade e mortalidade.

Um efeito adverso da clampagem do pedículo hepático é a isquemia do 
órgão, que juntamente com a sua reperfusão determina a lesão de isquemia e reperfusão (LIR). Até um certo limite, os pacientes irão responder melhor aos danos causados pela LIR do que aos causados por hemorragias extensas e múltiplas transfusões. Ela resulta em insuficiência microcirculatória, seguida de morte celular, de modo que proteger os hepatócitos desse evento é importante para a cirurgia de fígado. Vários mecanismos têm sido propostos para explicar a LIR, mas a sua fisiopatologia ainda não é inteiramente clara $7,20,27,29,30$.

A apoptose é um tipo especial de morte celular, onde a célula é estimulada para desencadear mecanismos que culminarão com a sua própria morte. Por isso também é conhecida como suicídio celular ${ }^{8}$. Ela parece ser importante em situações de stress oxidativo e como um mecanismo de morte celular na $\operatorname{LIR}^{12,25}$.

A clampagem intermitente do pedículo hepático, em que períodos curtos de clampagem são intercalados com reperfusão, pode ser uma forma de minimizar o dano celular, porque impede isquemia quente prolongada ${ }^{1,4,28}$.

Este estudo teve como objetivo analisar a LIR hepática após clampagem contínua ou intermitente do pedículo hepático, utilizando a quantificação da apoptose no fígado como marcador. Dessa forma, diferentes níveis de indução de apoptose poderiam demonstrar a superioridade de um método sobre o outro, garantindo maior proteção ao parênquima hepático.

\section{MÉTODOS}

Vinte coelhos albinos adultos machos (Oryctolagus cuniculus) com e peso médio de 2218 $\pm 404 \mathrm{~g}$ (Fazenda Veterinária em Igarapé, MG, Brasil) foram submetidos à quarentena clínica, alojamento, avaliação individual, com fornecimento de água e ração ad libitum. Os animais foram divididos aleatoriamente em três grupos: controle $(n=5)$, isquemia contínua $(n=8)$ e isquemia intermitente $(n=7)$. Todos os procedimentos foram previamente aprovados pelo Comitê de Ética em Experimentação Animal da Universidade Federal de Minas Gerais (Certificado de Aprovação n ${ }^{\circ}$ 170/2006) e obedeceram a padrões internacionais.

O jejum pré-operatório foi de 12 horas para sólidos. Não houve jejum para líquidos. Os animais foram submetidos a dois procedimentos cirúrgicos, definidos como operação inicial e operação final, realizados com um intervalo de seis horas. Anestesia, operações e eutanásia (realizada imediatamente após a segunda operação) foram realizadas seguindo o protocolo publicado em nossa instituição 22 .

Os animais nos grupos experimentais de isquemia foram submetidos à laparotomia, biópsia hepática, isquemia contínua ou intermitente durante 60 minutos e à nova biópsia hepática imediatamente após a reperfusão; a seguir, eram acomodados em gaiolas para recuperação pós-operatória. Seis horas após a operação inicial, eles foram anestesiados e submetidos à outra laparotomia com biópsia hepática, seguida de eutanásia. As biópsias foram obtidas por meio de incisão em cunha do lobo direito e punção usando kit comercial Hepafix ${ }^{\circledR}$. Os momentos das coletas das biópsias foram nomeados em sequências como T1, T2 e T3. A clampagem do pedículo hepático foi feita como descrito por Pringle ${ }^{24}$ utilizando um laço e um anel de látex, e a intermitência foi obtida alternando períodos de 12 minutos de isquemia com três minutos de reperfusão.

\section{Morfometria}

Os fragmentos hepáticos foram colocados em frascos individuais identificados contendo $10 \%$ de formaldeído tamponado e processados rotineiramente com inclusão em parafina, corte e coloração com hematoxilina-eosina (HE) ou TUNEL ${ }^{14}$ para detectar a fragmentação do genoma in situ / caracterização da apoptose.

Foi utilizado um kit comercial para detecção in situ da fragmentação do genoma (FragEl Klenow DNA fragmentation Detection Kit - QIA21 no catálogo, Calbiochem/Oncogene, disponível no sítio da web http:// calbiochem.com/), seguindo o protocolo especificado pelo fabricante. O tecido foi desparafinizado por imersão em xilol e álcool em diluições decrescentes e em seguida lavado duas vezes com água destilada. A peroxidase endógena foi inativada pela cobertura das secções com peróxido de hidrogénio a $3 \%$ durante cinco minutos. 0 tecido foi então lavado em PBS e imerso em solução de tampão de equilíbrio. Os cortes foram cobertos com TdT (transferase terminal de deoxinucleotídeos), UTP e digoxigenina e colocados em atmosfera úmida a $37^{\circ} \mathrm{C}$ durante duas horas; em seguida foram incubados durante 10 minutos em tampão de lavagem e parada. A seguir, foram colocados em uma câmara umidificada à $37^{\circ} \mathrm{C}$ durante 18 horas (incubação overnight) com um conjugado anti-digoxigenina peroxidase. As lâminas foram novamente imersas em PBS, e, em seguida imersas durante seis minutos em diaminobenzina (revelação da reação), lavada com água destilada, coradas com o corante metil green e montadas para avaliação microscópica. As lâminas foram examinadas ao microscópio óptico e imagens dos campos histológicos foram digitalizadas e analisadas com um software de morfometria (UTHSCSA Image Tool para Windows ${ }^{\circledR}$ ) a fim de obter o índice apoptótico.

Cada corte histológico com a coloração HE teve campos aleatórios capturados em formato digital em aumento de 400x. Foram feitas, pelo mesmo observador, as contagens dos hepatócitos em apoptose e dos hepatócitos totais em cada campo. As células foram consideradas como apoptóticas quando apresentavam, pelo menos, três dos seguintes achados: 1) anoiquia (retração celular com perda da adesão com as células vizinhas); 2) condensação citoplasmática; 3) condensação nuclear (condensação da cromatina nuclear, às vezes permeando a parte superior da membrana nuclear e exibindo figuras de "crescentes"; 4) fragmentação nuclear; 5) formação de corpos apoptóticos (Figura 1). 


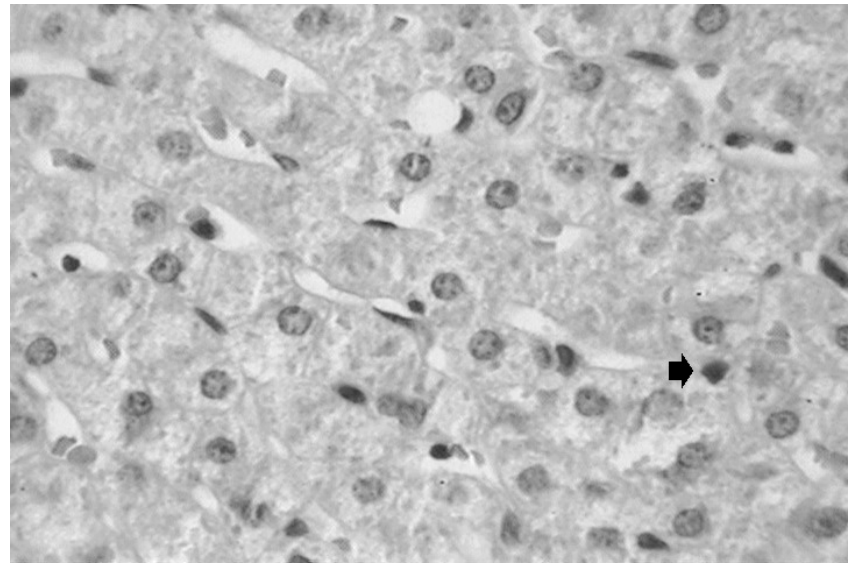

FIGURA 1 - Identificação de apoptose em um campo microscópico digitalizado. A seta aponta uma célula apoptótica com condensação nuclear e anoiquia. Hematoxilina-eosina, 400x

A quantificação da apoptose foi realizada por teste cego por um único observador. O índice apoptótico (IA) foi calculado pela seguinte fórmula:

\section{IA = (hepatócitos apoptóticos / hepatócitos totais) $\times 100$}

O coeficiente de variância (CV) foi utilizado para determinar o número mínimo de campos de visão necessariamente analisados a fim de obter a significância estatística. O método consistiu em obter sub-grupos de amostragem menor entre 100 campos diferentes de uma lâmina corada com HE escolhida aleatoriamente na amostra total. Sub-grupos foram escolhidos aleatoriamente com um número crescente de campos e os coeficientes de variância obtidos para cada sub-grupo foram traçados num gráfico, onde a curva foi obtida. Assim, o número mínimo de campos representativos foi encontrado quando o aumento do número de campos não resultou na redução significativa do valor do CV, que ocorreu após a contagem de 12 campos, como observado na Figura 2.

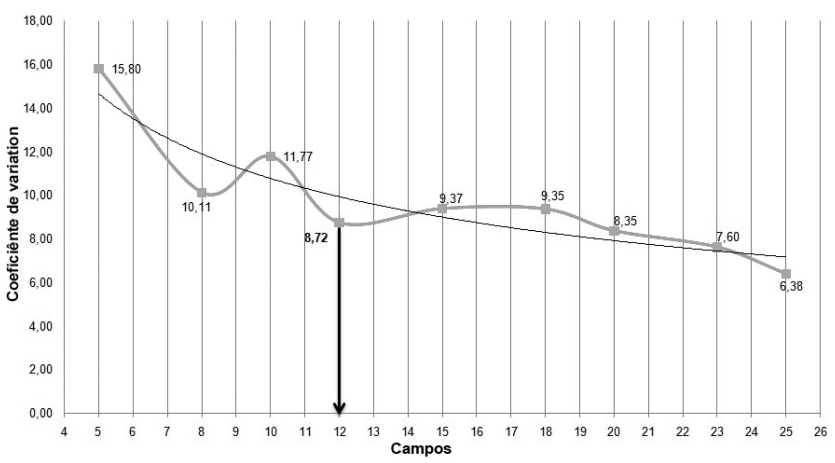

FIGURA 2 - Resultado da análise para determinação do número mínimo de campos representativos (com curva de tendência)

A validação dos resultados obtidos com a análise morfométrica das lâminas coradas com HE foi feito com reação de TUNEL em lâminas aleatoriamente selecionadas na proporção de $20 \%$ de todas as lâminas, uniformemente distribuídas pelos diferentes grupos. Estas lâminas foram submetidos à reação de TUNEL como descrito anteriormente. Os campos com ampliação de 400X foram digitalizados do mesmo modo como descrito para as lâminas coradas com $\mathrm{HE}$.

\section{Análise estatística}

Os dados obtidos neste estudo foram classificados como variáveis contínuas paramétricas e foram apresentados como média \pm desvio-padrão. O teste de Kolmogorov-Smirnov foi utilizado para verificar a normalidade da amostra e do teste t de Student com amostras independentes (entre os grupos) e amostras emparelhadas (análise intra-grupo). A significância foi considerada quando os valores de $\mathrm{p}$ foram inferiores a 0,05 . SPSS ${ }^{\circledR}$ e GraphPad Prism ${ }^{\circledR}$ para Windows ${ }^{\circledR}$ eram do software utilizado.

\section{RESULTADOS}

Todos os animais demonstraram comportamento normal durante a realização do estudo, sem manifestações clínicas de doença. Não houve necessidade de substituição de qualquer animal. Não houve complicações pré, trans ou pós-operatórias. Cerca de três a quatro horas após a operação inicial, os animais estavam acordado e em movimento.

\section{Quantificação de apoptose}

As médias dos IA obtidos para diferentes grupos e tempos apresentaram distribuição normal, de acordo com o teste de Kolmogorov-Smirnov, que apresentou distância $=0.1746(p>0.10$, alfa=0.05).

A distribuição das médias de IA ocorreu com uniformidade do índice no grupo controle e elevação após a intervenção nos grupos de isquemia, que retornou a índices próximos dos iniciais após seis horas de reperfusão (Tabela 1 ).

\begin{tabular}{|l|c|c|c|}
\hline Médias & T1 (0) & T2 (1h) & T3 $(6 h)$ \\
\hline Grupo 1 (controle) & 7,32 & 7,36 & 7,32 \\
\hline Grupo 2 (isquemia contínua) & 7,77 & 8,78 & 7,83 \\
\hline Grupo 3 (isquemia intermitente) & 7,93 & 8,64 & 7,79 \\
\hline
\end{tabular}

TABELA 1 - Médias dos índices apoptóticos nos grupos, de acordo com o momento do experimento (tempo)

No grupo controle não houve diferença entre os IA em nenhum momento do protocolo. Nos grupos de isquemia encontrou-se diferença no final da isquemia em relação ao início do experimento, e a reperfusão foi capaz de alterar o IA em relação ao final da isquemia, em redução para valores iguais aos encontrados no início do experimento. O resultado da análise intragrupo está sumarizada na Tabela 2. 


\begin{tabular}{|c|c|c|c|c|c|}
\hline \multirow[b]{2}{*}{ Grupo } & \multirow[b]{2}{*}{ Par } & \multicolumn{3}{|c|}{ Diferença pareada } & \multirow[b]{2}{*}{ Valor $p$} \\
\hline & & Média & $\begin{array}{l}\text { Desvio } \\
\text { padrão }\end{array}$ & $\begin{array}{l}\text { Erro } \\
\text { padrão }\end{array}$ & \\
\hline \multirow{3}{*}{$\begin{array}{l}\text { Grupo } 1 \\
\text { Controle }\end{array}$} & IA1 - IA2 & $-0,38$ & 0,18 & 0,81 & 0,666 \\
\hline & IA 2 - IA3 & 0,46 & 0,22 & 0,98 & 0,664 \\
\hline & IA 1 - IA3 & 0,08 & 0,17 & 0,74 & 0,920 \\
\hline \multirow{3}{*}{$\begin{array}{c}\text { Grupo } 2 \\
\text { Isquemia contínua }\end{array}$} & IA 1 - IA2 & $-1,01$ & 0,56 & 0,20 & $0,001^{*}$ \\
\hline & IA2 - IA3 & 0,95 & 0,53 & 0,19 & $0,002^{*}$ \\
\hline & IA 1 - IA3 & $-0,57$ & 0,59 & 0,21 & 0,794 \\
\hline \multirow{3}{*}{$\begin{array}{c}\text { Grupo } 3 \\
\text { Isquemia intermitente }\end{array}$} & IA 1 - IA2 & $-0,71$ & 0,47 & 0,18 & $0,007^{*}$ \\
\hline & IA 2 - IA3 & 0,85 & 0,79 & 0,30 & $0,030^{*}$ \\
\hline & IA1 - IA3 & 0,14 & 0,39 & 0,15 & 0,375 \\
\hline
\end{tabular}

TABELA 2 - Análise pareada dos índices apoptóticos

A qualificação pelo método de TUNEL para esses resultados obtidos foi feita por amostragem, onde para um mesmo animal obteve-se campos TUNEL antes e após a isquemia e observou-se a presença de coloração variando de fraca para forte (Figura 3).

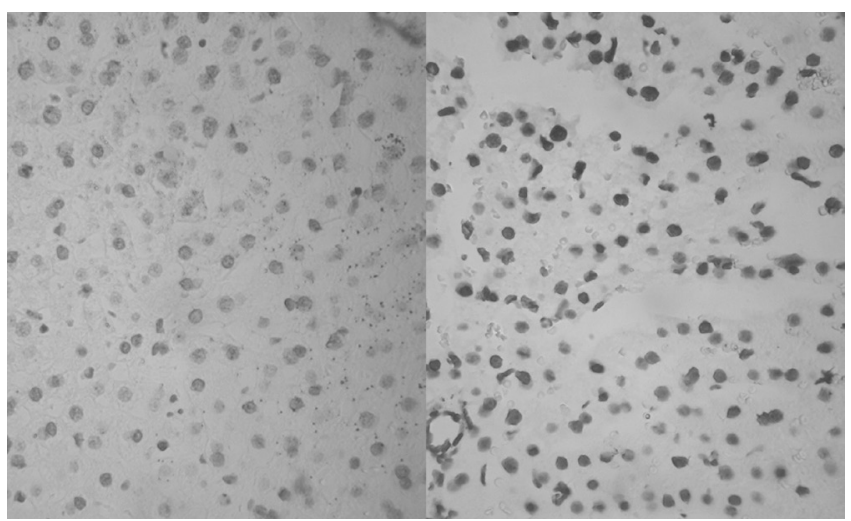

FIGURA 3 - Campo microscópico mostrando coloração por TUNEL antes e depois da isquemia, qualificando o resultado da contagem para obtenção do índice apoptótico (TUNEL, 400x).

A análise inter-grupos demonstrou que os IA dos grupos eram iguais antes da isquemia. Ela produziu diferença entre os índices de ambos os grupos em relação ao grupo controle. Os resultados estão sumarizados na Tabela 3 que mostra, também, que não houve diferença de IA entre os grupos e controles após a reperfusão.

\section{DISCUSSÃO}

Os trabalhos disponíveis na literatura que estudam a LIR hepática com modelos animais são numerosos e focam em diferentes variáveis, incluindo o modelo animal, método e tempo de isquemia, isquemia intermitente, pré-condicionamento isquêmico e condicionamento isquêmico remoto ${ }^{18,19}$. Este estudo teve como objetivo identificar, entre dois métodos de clampagem do pedículo hepático (métodos que apresentam diferenças técnicas, com vantagens e desvantagens em ambos os lados), qual produz menos dano ao fígado.

Sabe-sequea LIR hepáticaé consequência de isquemia normotérmica ou hipotérmica seguida por reperfusão. A disfunção tecidual é secundária a esses eventos. A duração da isquemia influencia o grau de lesão, embora muitas das lesões apareçam na fase de reperfusão $0^{5,718,20,29}$.

Existem algumas ferramentas para avaliar o grau de dano provocado pela LIR, incluindo marcadores séricos com graus variados de especificidade, marcadores específico de tecido, morfometria e imunoistoquímica ${ }^{11,28}$. Não há abordagem de consenso de qual método é melhor para o clampeamento do pedículo hepático e muitos autores apresentam diferentes resultados ${ }^{4,21,23}$. O Instituto Cochrane de Revisões Sistemáticas publicou um artigo em 2007 que mostra a evidência de que a clampagem intermitente é segura, mas não diminui a morbidade do procedimento, sem qualquer evidência clínica aparecendo até aquele momento ${ }^{15}$.

$O$ uso de coelhos permite o estudo da LIR em um modelo de isquemia hepática total, ao contrário de outros roedores, em que os modelos podem ser usados apenas com clampagem hepática parcial ou subtotal (que não reflete as situações que ocorrem na clínica, em muitos casos) ou com a adição de complicações técnicas. Observou-se que esses animais toleram a isquemia por 60 minutos com baixa mortalidade e submetêlos à isquemia intermitente não produz mortalidade ${ }^{18}$. Diferentes modelos de isquemia intermitente têm sido descritos e aqui foi escolhido um deles, considerandose que após 10 a 12 minutos de isquemia, três a cinco minutos de reperfusão são suficientes para restaurar a oxigenação tecidual intra-hepática ${ }^{6,23,28}$. Neste estudo, a diferença entre o tempo de isquemia total nos grupos contínuo e intermitente (60 versus 48 minutos) ocorreu para mimetizar as situações clínicas reais experimentadas durante os procedimentos cirúrgicos ${ }^{4}$.

A apoptose é provocada por isquemia, não só no fígado, mas também em outros órgãos. Diferentes trabalhos mostraram aumento na intensidade da apoptose secundária à LIR e descobriram que a sua ativação ocorre durante a reperfusão precoce, isto é, apenas após o final da isquemia no fígado. Finalmente, a apoptose parece ser um modo predominante de morte celular em LIR lesão hepática durante a isquemia quente ${ }^{12,13}$.

A utilização de TUNEL para avaliar a apoptose em células do fígado e em situações de isquemia de vários órgãos é um consenso na literatura ${ }^{3,17,26}$. Em um modelo experimental em ratos Sprague-Dawley, Baier et al. ${ }^{3}$ descobriram que, após 15 minutos de isquemia, houve indução de apoptose detectável por TUNEL em tempos de reperfusão que variaram de 30 minutos a duas horas.

O bloqueio da apoptose foi proposto ${ }^{10}$ para proteção celular durante a isquemia e reperfusão hepáticas. Crenesse et al. ${ }^{9}$ provocou isquemia intermitente em um modelo experimental, resultando em redução de apoptose com diferença significativa na expressão de TUNEL entre esse tipo de isquemia, isquemia contínua e controles, e apresentou o IA como um método eficiente para quantificar, em valores contínuos, a apoptose no fígado.

Existem resultados variáveis em estudos quantitativos mostrando $5-80 \%$ de apoptose após isquemia ${ }^{10,16,17}$. Estudos isolados, no entanto, não 
mostraram apoptose ${ }^{2}$ ou encontraram necrose como o achado dominante ${ }^{20}$. Nesses estudos, no entanto, o tempo de isquemia pareceu ser excessivo.

Os IA avaliados por análise pareada nos grupos isquemia contínua e intermitente, elevaram-se após o período de isquemia e retornaram aos valores iniciais após seis horas de reperfusão. Independentemente, os IA entre os grupos de isquemia não mudaram de um grupo para outro, de modo que os IA nesse estudo não demonstraram diferença entre os métodos de clampagem. Isso não aconteceu com outros autores que relataram diferença entre IA na isquemia contínua e intermitente ou citaram diferença, mas não quantificada9 .

Finalmente, o último aspecto a discutir é o uso de coloração HE padrão, sem utilização de reação histoquímica e imunoistoquímica para quantificar a apoptose, que encontra subsídios na literatura ${ }^{17,26}$. A facilidade de utilização, após treinamento adequado, proporciona baixo custo uma vez que reduz a quantidade de reações histoquímicas realizadas.

\section{CONCLUSÃO}

Baseados nos resultados desse estudo, os autores concluem que há apoptose após clampagem do pedículo hepático, mas não houve diferença em sua expressão entre os métodos contínuo ou intermitente; portanto não se pode determinar, com essa ferramenta, superioridade de um método sobre o outro.

\section{AGRADECIMENTOS}

Os autores agradecem à Fundação de Amparo à Pesquisa de Minas Gerais (FAPEMIG) por seu suporte financeiro, e também à Universidade de Ciências da Saúde de Alagoas - UNCISAL, Faculdade de Medicina da Universidade Federal de Minas Gerais (Prof. Dr. Andy Petroianu, Dr. Guilherme Medrado e Dra . Fernanda Mendes) e ao Departamento de Patologia (Dr. Antenor Leal) da Santa Casa de Maceió.

\section{REFERÊNCIAS}

1. Abdalla EK, Noun R, Belghiti J. Hepatic vascular occlusion: which technique? Surg Clin North Am. 2004 Apr;84(2):563-85.

2. Altunkan A, Aydin O, Ozer Z et al. Anti-apoptotic effect of succinyl gelatine in a liver ischaemia-reperfusion injury model (Bcl-2, Bax, Caspase 3)? Pharmacol Res. 2002 Jun;45(6):485-9.

3. Baier PK, Baumgartner U, Wolff-Vorbeck G et al. Hepatocyte proliferation and apoptosis in rat liver after liver injury. Hepatogastroenterology. 2006 Sep-Oct; 53(71):747-52.

4. Belghiti J, Noun R, Malafosse R et al. Continuous versus intermittent portal triad clamping for liver resection: a controlled study. Ann Surg. 1999 Mar;229(3):369-75.

5. Bismuth H, Castaing D, Garden OJ. Major hepatic resection under total vascular exclusion. Ann Surg. 1989 Jul;210(1):13-9.

6. Brooks AJ, Hammond JS, Girling K et al. The effect of hepatic vascular inflow occlusion on liver tissue $\mathrm{pH}$, carbon dioxide, and oxygen partial pressures: defining the optimal clamp/release regime for intermittent portal clamping. J Surg Res. 2007 Aug;141(2):247-51.

7. Castro e Silva Jr Od, Centurion S, Pacheco EG et al. Aspectos básicos da lesão de isquemia e reperfusão e do pré-condicionamento isquêmico. Acta Cirurgica Brasileira. 2002;17:96-100.

8. Cohen JJ. Apoptosis. Immunology today. 1993;14(3):126-30.

9. Crenesse $D$, Laurens $M$, Gugenheim J et al. Intermittent ischemia reduces warm hypoxia-reoxygenation-induced JNK(1)/SAPK(1) activation and apoptosis in rat hepatocytes. Hepatology. 2001 Nov;34(5):972-8.

10. Cursio R, Auberger $P$, Gugenheim J. Inhibition of apoptosis: a new therapeutic approach to prevent liver ischemia-reperfusion lesions? Gastroenterol Clin Biol. 2000 Jun-Jul;24(6-7):607-8.

11. Cursio R, Filippa N, Miele $C$ et al. Fas ligand expression following normothermic liver ischemia-reperfusion. J Surg Res. 2005 May 1;125(1):30-6

12. Feldmann G. Liver apoptosis. J Hepatol. 1997;26 Suppl 2:1-11.

13. Gao W, Bentley RC, Madden JF et al. Apoptosis of sinusoidal endothelial cells is a critical mechanism of preservation injury in rat liver transplantation. Hepatology. 1998 Jun;27(6):1652-60.

14. Gavrieli Y, Sherman Y, Ben-Sasson SA. Identification of programmed cell death in situ via specific labeling of nuclear DNA fragmentation. J Cell Biol. 1992 Nov;119(3):493-501.

15. Gurusamy KS, Kumar Y, Sharma D et al. Methods of vascular occlusion for elective liver resections. Cochrane Database Syst Rev. 2007(4):CD006409.

16. Helling TS, Edwards CA, Helling TS, Jr et al. Hepatic apoptotic activity following transient normothermic inflow occlusion and reperfusion in the swine model. J Surg Res. 1999 Sep;86(1):70-8.

17. Jawan B, Goto S, Pan TL et al. The protective mechanism of magnolol, a Chinese herb drug, against warm ischemia-reperfusion injury of rat liver. J Surg Res. 2003 Apr;110(2):378-82.

18. Kanoria S, Glantzounis G, Jalan R et al. A model to study total hepatic ischemia-reperfusion injury. Transplant Proc. 2004 Nov;36(9):2586-9.

19. Kanoria S, Jalan R, Davies NA et al. Remote ischaemic preconditioning of the hind limb reduces experimental liver warm ischaemia-reperfusion injury. Br J Surg. 2006 Jun;93(6):762-8.

20. Meguro $\mathrm{M}$, Katsuramaki T, Kimura $\mathrm{H}$ et al. Apoptosis and necrosis after warm ischemia-reperfusion injury of the pig liver and their inhibition by ONO-1714. Transplantation. 2003 Mar 15;75(5):703-10.

21. Nieuwenhuiij VB, de Bruijn MT, Schiesser Metal.Ischemic preconditioning and intermittent ischemia preserve bile flow in a rat model of ischemia reperfusion injury. Dig Dis Sci. 2007 May;52(5):1159-67.

22. Oliveira HP. Protocolos anestésicos comumente utilizados em animais de pequeno porte. 2006 [cited 200819 out]; Available from: http:// www.ufmg.br/bioetica/cetea/index.php?option=com_content\&task= view\&id $=22 \&$ Itemid $=35$

23. Petrowsky H, McCormack L, Trujillo M et al. A prospective, randomized, controlled trial comparing intermittent portal triad clamping versus ischemic preconditioning with continuous clamping for major liver resection. Ann Surg. 2006 Dec;244(6):921-8; discussion 8-30.

24. Pringle JH. V. Notes on the Arrest of Hepatic Hemorrhage Due to Trauma. Ann Surg. 1908 Oct;48(4):541-9.

25. Sasaki H, Matsuno T, Nakagawa K et al. Induction of apoptosis during the early phase of reperfusion after rat liver ischemia. Acta Med Okayama. 1997 Dec;51(6):305-12.

26. Schauer RJ, Gerbes AL, Vonier D et al. Glutathione protects the rat liver against reperfusion injury after prolonged warm ischemia. Ann Surg. 2004 Feb;239(2):220-31.

27. Serracino-Inglott F, Habib NA, Mathie RT. Hepatic ischemia-reperfusion injury. Am J Surg. 2001 Feb;181(2):160-6.

28. van Wagensveld BA, van Gulik TM, Gelderblom HC et al. Continuous or intermittent vascular clamping during hemihepatectomy in pigs: hyaluronic acid kinetics in the assessment of early microvascular liver damage. Eur J Surg. 2000 Mar;166(3):255-61.

29. Vollmar B, Glasz J, Leiderer R et al. Hepatic microcirculatory perfusion failure is a determinant of liver dysfunction in warm ischemiareperfusion. Am J Pathol. 1994 Dec;145(6):1421-31.

30. Vollmar B, Richter S, Menger MD. Liver ischemia/reperfusion induces an increase of microvascular leukocyte flux, but not heterogeneity of leukocyte trafficking. Liver. 1997 Apr;17(2):93-8. 